\title{
SWARRECOG- THE EUPHONY AIDE
}

\author{
Shreyash Bardia, Krishnakumar Natthani, Sachin Rawool and Sakshi Kukreja
}

\author{
Savitribai Phule Pune University, Pune, India
}

\begin{abstract}
Almost everyone is interested in learning music. But due to lack of knowledge about music, they are unable to make it happen. It is where this system comes into picture-The Swar Reorganization System will help musicians as well as music enthusiasts to have notations of a song on-the-go which in turn will elevate their music literacy.

The need as you know is we live in a digital era where almost each and everything we use to interact with each other is digitalized. The main motive behind it being computerized is that it will help musicians and music enthusiast with an opportunity to record, learn and share their ideas whenever and wherever required i.e. providing portability with it which as we all know is the most vital and required function of a system these days, as your system moves with you.
\end{abstract}

Keywords: SWARRECOG, sārangüin

\section{INTRODUCTION}

Indian art music encapsulates two music traditions of the ScoreCloud, MuseScore and many more are existing Indian subcontinent: Hindustani music (also known as systems which helps users to convert music into notations. North Indian music), prominent in the northern regions of But these all providers transcript into western notation. India, Pakistan, Nepal, Afghanistan and Bangladesh; and Whereas, here what we are aiming to do is the same in Carnatic music, widespread in the southern regions of Hindustani Bhatkhande Paddhat (method). As well as peninsular India and Sri Lanka. In both Hindustani and these all systems are available only for desktop users and Carnatic music, the rāg is the fundamental melodic user base is also overwhelming, after our brief survey it framework upon which the music is built, and the $t a \bar{l}($ tāla came to our attention that no such system exists for in the Carnatic music tradition) provides the rhythmic android platform and hence we have taken this opportunity framework.

to venture in this domain and develop a masterpiece which Indian art music is basically heterophonic, with the main will be available to its user on the go. melody sung or played by the lead artist. Often, an instrument provides a melody accompaniment by closely following the melody rendered by the lead artist. A typical The SwarRecogis about taking in a MIDI file, reading it arrangement in a performance of Indian art music consists by bytes and interpreting the information inside, and then of a lead performer (occasionally a duo), a melody converting it into a readable musical score. Every MIDI accompaniment provided by harmonium or sārangīin file follows a format, so it is possible to make an Hindustani music and by violin in Carnatic music, a application to access all pertaining information inside. rhythm accompaniment usually provided by tabläin Accessing MIDI file will be done by reading the file word Hindustani music and mrdangam in Carnatic music and a per word to find the pattern. The data read will be in object constantly sounding drone in the background. The drone file so it is passed by reference. After the data is read, it sound, which is typically produced by the tannp $\bar{r} \bar{a}$, is the will be sent immediately to be processed by another only component that adds a harmonic element to the method and the graphical representation in Hindustani performance.[4]

The seven solfège symbols ( $\mathrm{Sa}, \mathrm{Re}, \mathrm{Ga}, \mathrm{Ma}, \mathrm{Pa}$, Dha and $N \bar{i}$ in short-form) used in Indian art music are called swars. With the exception of Sa (also referred toasSadja) and $\mathrm{Pa}$ (also referred to as Pancham, fifth with respect to $\mathrm{Sa}$ ), every other swar has two or three variations, where each variation is either a komal (flat), śudh (unmodified, literally means pure) or tivvr (sharp) of the basic swar and has a specific function in a rāg rendition.

MIDI (Musical Instrument Digital Interface) is technical standard that describes a protocol, digital interface and connectors and allows a wide variety of electronic musical instruments, computers and other related devices to connect and communicate with one another. A single MIDI link can carry up to sixteen channels of information, each of which can be routedto a separate device and carries event messages that specify notation, pitch and velocity, control signals for parameters such as volume, vibrato, audio panning,cues and clock signals that set and synchronize tempo between multiple devices. notation will be created.

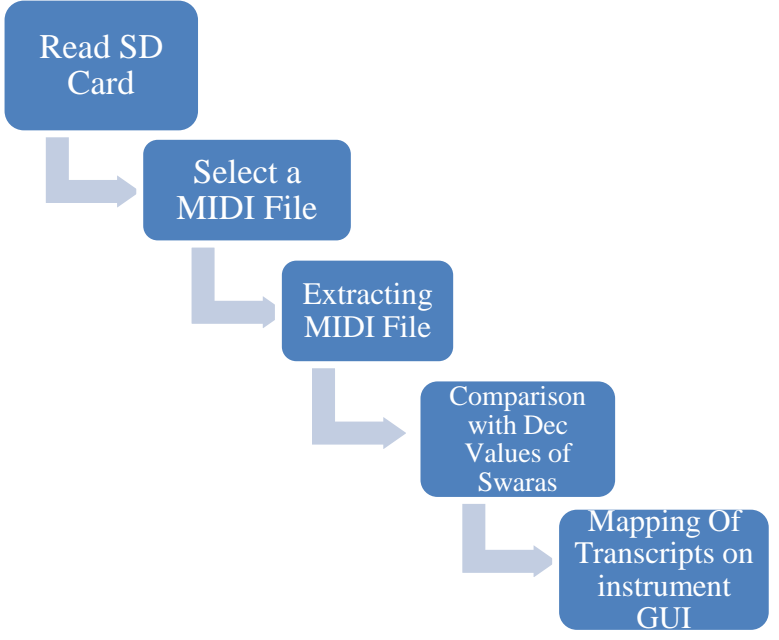

Fig 2.1 Flow Diagram 
Figure 2.1 clearly shows the flow that the user might do with the application. The user will start by giving an input The method will next continue the painting of the score by to the application regarding the location of the MIDI file. drawing Synthesizer and highlighting the notes being This can be done by using Play Button provided on the played. There is a different color used for both hands to GUI, which opens the file browser, where the user can differentiate,

browse to the location of the file and choose it. After the 1. Keys highlighted in Blue represent the left hand file path is entered, the user must check the file validity. If Progression

the path provided refers to a MIDI type 2, a non MIDI file, 2. Keys highlighted in Grey represent the Right hand Lead or a non-existent file, an error dialog box will appear to notify the user of this failure. If a correct path has been confirmed, the user will be enabled of running the transcription process. The application will then run the process of transcripting MIDI and give result to the user.

\section{METHODOLOGY}

As the file is selected it is checked for its validity, once the file selected is a termed as a valid MIDI file it will continue by checking and drawing the time signature and pitches. The pitch will again be checked for its individual accidentals, sharp or flat. Since there are 7 natural pitch, there are 5 accidentals in total. Sa, re, Re, ga, Ga, Ma, ma, $\mathrm{Pa}$, dha, Dha, ni, Ni. For example, $\mathrm{Sa}=60$, $\mathrm{re}=61, \mathrm{Re}=$

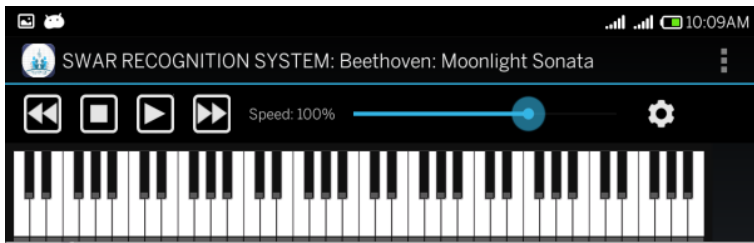

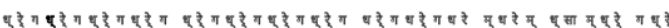

Fig.2.2 Instrument GUI

$62, \mathrm{ga}=63, \mathrm{Ga}=64, \mathrm{Ma}=65, \mathrm{ma}=66, \mathrm{~Pa}=67, \mathrm{dha}=68$, Below the GUI, the transcriptions of song are played in Dha $=69, \mathrm{ni}=70, \mathrm{Ni}=71$. The pitch number will be sync which will help the user to know which note is divided 12 and checked if the remainder is $1,3,6,8,10$. currently being played.

Those are the numbers that is a sharp or a flat, while the rest are natural pitch.

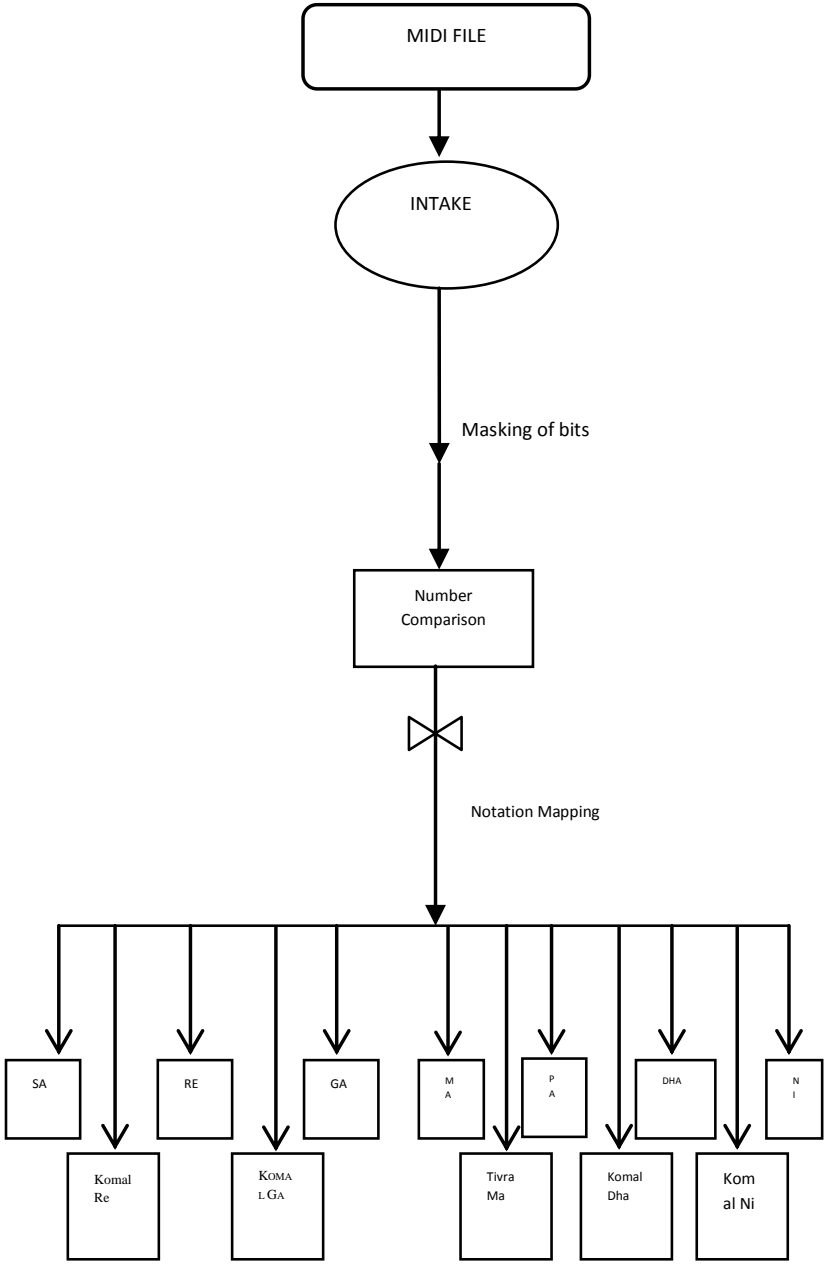

There is also a media control bar provided as can be seen in Fig.2.2 from where user can play, stop, reverse, forward the current song.

Another important part of this media bar is the speed control interface which allows user to slow down or speedup the current song allowing him/her to practice according to their tempo. The speed bar can slow down upto $10 \%$ and increase the speed up to $150 \%$ of the original tempo allowing user a wide range of choice.

\section{ADVANTAGES}

1. The first and foremost advantage of this system is that it enables an independent learning environment.

2. A feast for musician and music enthusiasts as transcription in Hindustani Notations.

3. Portability, as an application for mobile platform, user can use it at his will wherever he pleases to do so.

\section{CONCLUSION}

Since music has become an inseparable part of human life, Musical Instrument Digital Interface (MIDI) has been a help to musician and music lovers. Not everyone who is willing to learn music is able to do so due to circumstances and issues. SwarRecog taking the advantage of MIDI format enable the individual to do so by providing the user with the transcription of the song in Hindustani notation allowing him to understand the composition, learn it and reproduce it at his will. This paper shows that MIDI file can be converted into musical notation because all the information needed is contained inside the file itself. The application shows that the transcription is possible, as long as the file inputted is the correct MIDI type 0 or 1 file. 


\section{REFERENCES}

[1] RikipGinanjar and Ivan Iskandar "MIDI Conversion to Musical Notation" 2011 FirstInternational Conference on Informatics and Computational Intelligence.

[2] Carol L. Krumhansl "Cognitive foundation of musical pitch" Department of Psychology Cornell University.

[3] Gopala K. Koduri1, Joan Serra2, and Xavier Serra "Characterization of intonation in carnatic music by parameterizing pitch histograms" Music Technology Group, Universitat pompeuFabra, Barcelona, Spain, Artificial Research Institute (IIIACSIC), Bellaterra, Barcelona, Spain.

[4] Sankalp Gulati, AshwinBellur, Justin Salamon, Ranjani H.G., VigneshIshwar, Hema A Murthy and Xavier Serra" Automatic tonic identification in Indian art music".

[5] Justin Salamon, Sankalp Gulati and Xavier Serra "A multipitch approach to tonic identification in Indian classical music" Music technology group Universitat PompeuFabra, Barcelona, Spain. 